Original Research Article

\title{
Best technique for fixation of displaced transverse olecranon fractures: tension band wiring or plate fixation?
}

\author{
Lokesh Kumar Yogi, Gagandeep Mahi*, C. R. Thorat, Moti Janardhan Naik
}

Department of Orthopedics, Government Medical College and Hospital Aurangabad, Maharashtra, India

Received: 22 May 2020

Revised: 02 July 2020

Accepted: 03 July 2020

\section{*Correspondence:}

Dr. Gagandeep Mahi,

E-mail:drgaganmahi@gmail.com

Copyright: (C) the author(s), publisher and licensee Medip Academy. This is an open-access article distributed under the terms of the Creative Commons Attribution Non-Commercial License, which permits unrestricted non-commercial use, distribution, and reproduction in any medium, provided the original work is properly cited.

\begin{abstract}
Background: Fractures of olecranon are common fractures in upper limb. Tension band wiring (TBW) and plate fixation (PF) are mostly used techniques but choice is based on type of fracture and surgeon's preference.

Methods: A study assessed functional results in 28 patients that were enrolled after the clinical event of trauma has occurred. Patients were divided into two groups- Group (A) for TBW and Group (B) for PF; here gender, age and side of fracture were ignored. Post-operative functional outcome were evaluated by using the Mayo Elbow Performance (MEP) and the Disabilities of the Arm, Shoulder and Hand (DASH) score parameters.

Results: Mean (SD) union time as determined by postoperative radiographs was 8.5 (1.48) weeks for group (A) and 9 (2.08) weeks for group (B). Mean (SD) MEP score at 9 months in group (A) 84.28 (7.28) and 80.71 (10.92) in group (B). Mean (SD) DASH at 9 months in group (A) 12.2 (8.8) and 11.7 (10.4) in group (B). Complications were reported in group (A) 6 patient (42.85\%) out of 14 patients and in group (B) 1 patient (7.14\%) out of 14 patients.

Conclusions: The current study shows that there are no significant differences in functional outcome between both the study groups. Due to lesser complications, we recommend the plate fixation approach as the better choice for transverse displaced olecranon fractures. More large scale studies are required to further confirm our results.
\end{abstract}

Keywords: Transverse displaced olecranon fracture, TBW, PF

\section{INTRODUCTION}

Isolated olecranon fractures are $10 \%$ of upper limb fractures in adults. ${ }^{1}$ Proximal ulna fractures can classified through various types of classifications. ${ }^{2}$ Mayo classification (Figure 1) is most preferred for olecranon fractures, as this classification not only describes fracture anatomy, morphology but also fracture stability, therefore it have important role for decision making during fracture fixation. ${ }^{3,4}$
Conservative management is reserved only for elderly patients ${ }^{5}$ Most of the olecranon fractures involve articular surface, except triceps avulsion fractures. Therefore, anatomical reduction is required to prevent the arthritic changes in the elbow joint. According to many surgeons "Gold standard fixation method" for transverse intraarticular olecranon fracture is $\mathrm{TBW}^{6}$, but $\mathrm{PF}$ is best for Monteggia, comminuted, oblique olecranon fractures, and fractures with dislocation., ${ }^{2,7}$ Still a controversy exists between TBW and PF that which is the best fixation method for olecranon fracture. ${ }^{8}$ 


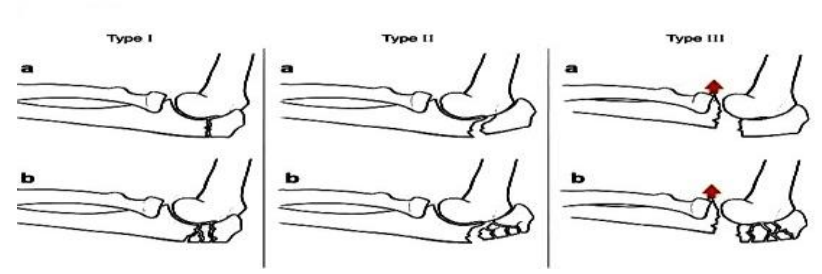

Figure 1: Mayo classification: type I undisplaced; type II displaced/stable; type III displaced/unstable; (a) simple, (b) comminuted.

\section{METHODS}

A study used existing data that have been recorded between July 2017 and October 2018 inclusive, 28 patients were matched for this study with following inclusion criteria's: closed transverse displaced olecranon fracture (Mayo type IIa and type IIIa) (figure 2), age at least 18 years or older and follow up at least 9 months. Patients were not included for this study if they had undisplaced olecranon fractures, comminuted olecranon fractures, pathological fractures and patients with polytrauma. Patient distribution in our study was divided into two groups, group (A) where 14 patients were treated with TBW and group (B) in which 14 patients were treated by PF; here gender, age and side of fracture were ignored.

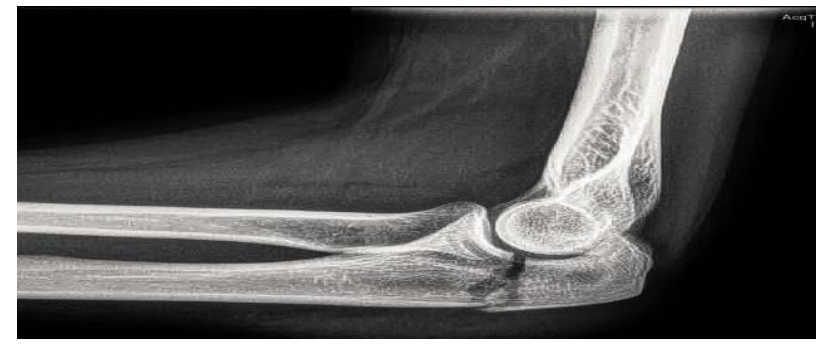

Figure 2: Transverse displaced olecranon fracture.

All operations were performed with the patients under axillary block (regional anaesthesia) with sedation and in lateral decubitus position. Both the procedures were done under tourniquet compression to reduce blood loss and the fracture was exposed through a posterior midline incision. Reduction was performed under fluoroscopic imaging using K-wires or bone clamps.

In TBW two K-wires (parallel 1.8 or $2.0 \mathrm{~mm} \mathrm{~K}$-wires) were passed from the tip of olecranon along the long axis of the ulna to the distal fragment, and passed across the anterior cortex of the ulna, then a transverse ulnar hole was drilled $3 \mathrm{~cm}$ distal to fracture site, a tension band wire passed through this drilled hole then formed figure of eight manner loop over the posterior surface of olecranon and tied (Figure 3). In PF a large midline incision were taken on the posterior surface of the proximal ulna for proper positioning of the plate (standard, long proximal, 8-hole or 12-hole) then fracture reduction was fixed with plate and screws (Figure 4).

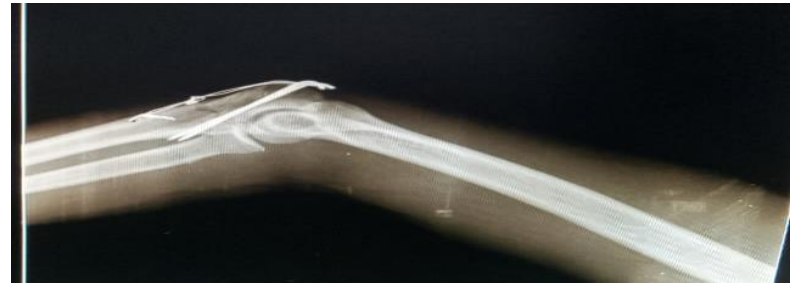

Figure 3: Olecranon tension band wiring.

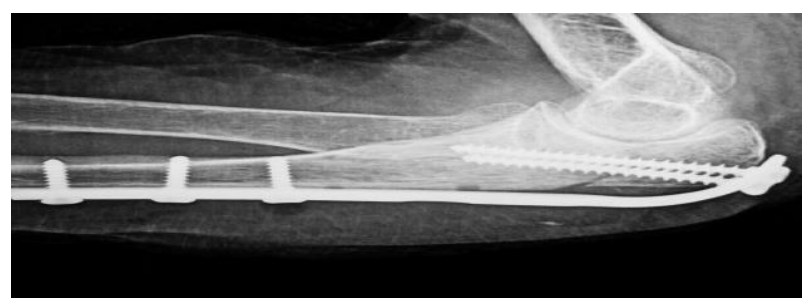

Figure 4: Olecranon platin.

The postoperative protocol included antibiotics administration for 48-72 hours; passive and activeassisted range of motion initiation at second postoperative day in group (A) patients and in group (B) at second week from the postoperative day. Routinely Follow-up were performed at 1 month, 2 months, 3 months, 6 months, 9 months, 12 months and thereafter at 1 year interval. At each follow clinical (MEP score and DASH) and radiological outcomes were evaluated. Average follow up was 9 months.

The functional outcome of this study was assessed by the following self-administered evaluation scales: the MEP Score $^{9}$ and the DASH. ${ }^{10}$

Data of this study was analysed with Wilcoxon rank test. If $p<0.05$ was counted as statistically significant $(S)$ and $\mathrm{p}>0.05$ was counted as statistically not significant (NS).

\section{RESULTS}

From the total of 28 patients who were included in the study, there were 15 males and 13 females with a mean (SD) age of 47.07 (11.03) years (range from 29 to 69 years). The frequency of fractures was higher in males till 50 years of age but altered in older decades towards females; the mean (SD) age of males was 39.53 (6.49) years and for females mean (SD) age was 55.76 (8.49) years.

Regarding the mechanism of injury, pattern was low energy trauma in 16 patients due to slip or simple fall onto the arm and high energy trauma in 12 patients (10 patients were road traffic accidents and 2 patients had fall from height).

Mean (SD) operative time periods for group (A) was 65.17 (5.93) minutes (range 60 to 75) and for group (B) 66.78 (5.85) minutes (range 60 to 80). Mean day of mobilization post-operatively was 2 nd day for group (A) 
and 14th day for group (B). Mean (SD) union time as determined by postoperative radiographs was $8.5(1.48)$ weeks (range 7-12) for group (A) and 9 (2.08) weeks (range 7-14) for group (B).

Mean (SD) Mayo Elbow Performance (MEP) score at 9 months in group (A) 84.28 (7.28) (range 70-95) and 80.71 (10.92) (range 60-95) in group (B). Therefore according to MEP 100 points scale, both groups having mean MEP scores in between range 75 to 89 , suggestive that both groups have 'Good category' functional outcomes. Mean (SD) DASH at 9 months in group (A) was 12.2 (8.8) and 11.7 (10.4) in group (B) (Table 1).

Table 1. Results of two comparative groups.

\begin{tabular}{|c|c|c|c|}
\hline Mean (SD) & $\begin{array}{l}\text { Group } \\
\text { (A) }\end{array}$ & $\begin{array}{l}\text { Group } \\
\text { (B) }\end{array}$ & P value \\
\hline $\begin{array}{l}\text { Operative } \\
\text { time(min) }\end{array}$ & $\begin{array}{l}65.17 \\
(5.93)\end{array}$ & $\begin{array}{l}66.78 \\
(5.85)\end{array}$ & 0.4760 \\
\hline $\begin{array}{l}\text { union } \\
\text { time(weeks) }\end{array}$ & $\begin{array}{l}8.5 \\
(1.48)\end{array}$ & $\begin{array}{l}9 \\
(2.08)\end{array}$ & 0.4702 \\
\hline MEP Score & $\begin{array}{l}84.28 \\
(7.28)\end{array}$ & $\begin{array}{l}80.71 \\
(10.92)\end{array}$ & 0.3182 \\
\hline $\begin{array}{l}\text { DASH } \\
\text { Score }\end{array}$ & $\begin{array}{l}12.2 \\
(8.8)\end{array}$ & $\begin{array}{l}11.7 \\
(10.4)\end{array}$ & 0.8918 \\
\hline
\end{tabular}

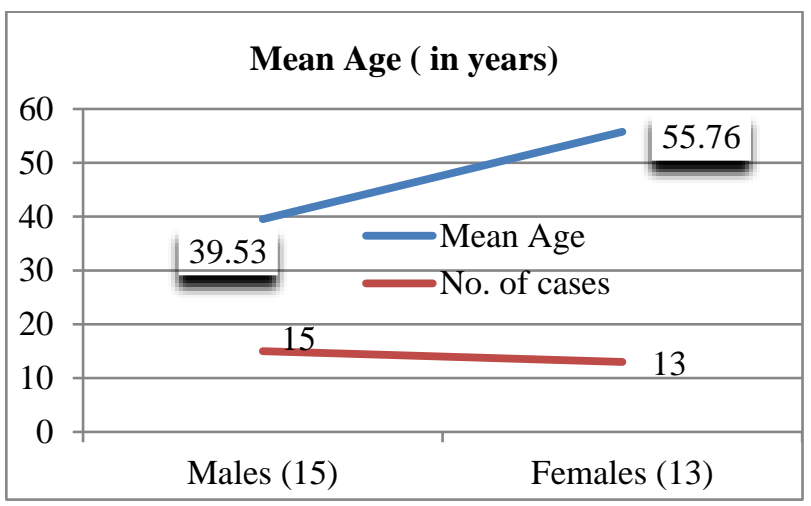

Figure 5: Graphic representation of the mean age of the male and female patients respectively.

The complications observed in group (A)- implant impingement in three patients and implant back out in three patients at 3 month follow-up, so implant removal was done for these six patients and above elbow slab was applied for 15 days, following which range of motion exercises were started; complications in group (B) were superficial infection in one patient at day 3 postoperatively for which antibiotic duration was extended for 7 days, when the infection settled the range of motion exercises were started. Overall complications that were noted in group (A) were 6 patients $(42.85 \%)$ out of 14 and in group (B) 1 patient $(7.14 \%)$ out of 14 . Hardware removal was more frequently performed in group (A): six patients versus zero patients for group (B). However, these differences didn't result in a significantly different outcome. Non-union and delayed union were not observed in both groups.

\section{DISSCUTION}

In this study, highest prevalence of olecranon fracture was reported in males less than 40 years and in females older age was observed. Similarly, Rommens et $\mathrm{al}^{1}$ reported that highest prevalence of olecranon fractures in males before 40 years of age and in females at an older age. Mechanism of injury in 16 patients out of 28 $(57.14 \%)$ was low energy trauma (slip or simple fall) and remaining had high energy trauma (road traffic accidents or fall from height). Similarly, Wolfgang et $a^{11}$ reported that 25 patients out of $45(55.55 \%)$ had fracture due to low energy trauma and similarly, Liu et al ${ }^{12}$ reported that 39 patients out of $62(62.90 \%)$ had fractures due to low energy trauma and remaining due to high energy trauma.

The comparative results of this study shows that there were no significant differences $(p>0.05)$ in the operative time periods ( $p$ value: 0.4760 ), union time ( $p$ value: 0.4702 ), MEP score ( $\mathrm{p}$ value: 0.3182 ), DASH ( $\mathrm{p}$ value: 0.8918 ); therefore, both groups have similar efficacy. Similarly, Tarallo et $\mathrm{al}^{13}$, Duckworth et al and Yi-Ming Ren et al in all their studies showed that there are no significant differences in functional outcome between both TBW and PF. ${ }^{14,15}$

The main complication following fixation of olecranon fractures in this study is related to impingement caused by hardware, mostly related to TBW but rare with PF. Duckworth et al, Yi-Ming Ren et al, Newman et al and Chan at el these all studies show impingement mostly related to TBW and less with $\mathrm{PF} .{ }^{14-17}$

\section{CONCLUSIONS}

In this retrospective study, both TBW and PF interventions have similar treatment benefits in transverse displaced olecranon fractures. Our study shows that there are no significant differences in operative time periods and functional outcome between both study groups. Due to less complication, we recommend the $\mathrm{PF}$ approach as the optimum choice for transverse displaced olecranon fractures. More large scale studies are required to further confirm our results.

Funding: No funding sources

Conflict of interest: None declared

Ethical approval: The study was approved by the institutional ethics committee

\section{REFERENCES}

1. Rommens PM, Küchle R, Schneider RU, Reuter M. Olecranon fractures in adults: factors influencing outcome. Injury. 2004;35:1149-57.

2. Nieto $\mathrm{H}$ et al. Proximal ulnar fractures in adults: a review of 163 cases. Injury. 2015;46:S18-23. 
3. Bernstein J, Monaghan BA, Silber JS, DeLong WG. Taxonomy and treatment: a classification of fracture classifications. J. Bone Joint Surg. Br. 1997;79:706-7.

4. Morrey BF. Current concepts in the treatment of fractures of the radial head, the olecranon, and the coronoid. Instr. Course Lect.1995;44:175-85.

5. Duckworth AD, Clement ND, McEachan JE, White TO, Court-Brown CM, McQueen MM. Prospective randomised trial of non-operative versus operative management of olecranon fractures in the elderly. Bone Joint J. 2017; 99-B (7):964-72.

6. Colton CL. Fractures of the olecranon in adults: classification and management. Injury. 1973;5(2):121-9.

7. Anderson ML, Larson AN, Merten SM, Steinmann SP. Congruent elbow plate fixation of olecranon fractures, J. Orthop. Trauma. 2007;21(6)386-93.

8. Duckworth AD, Clement ND, Aitken SA, CourtBrown CM, McQueen MM. The epidemiology of fractures of the proximal ulna. Inj.Int $\mathrm{J}$ Care Inj. 2012;43(3):343-6.

9. Cusick MC, Bonnaig NS, Azar FM, Mauck BM, Smith RA, Throckmorton TW. Accuracy and reliability of the Mayo Elbow Performance Score. J Hand Surg Am. 2014;39(6):1146-50.

10. Hudak PL, Amadio PC, Bombardier C. Development of an upper extremity outcome measure: the DASH (disabilities of the arm, shoulder and hand) [corrected]. The Upper Extremity Collaborative Group (UECG). Am J Ind Med. 1996;29:602-8.

11. Wolfgang $G$ et al. Surgical treatment of displaced olecranon fractures by tension band wiring. Clin Orthop Relat Res. 1987(224):192-204.
12. Liu QH et al. Randomized prospective study of olecranon fracture fixation: cable pin system versus tension band wiring. J Int Med Res. 2012;40(3):1055-66.

13. Tarallo L, Mugnai R, Adani R, Capra F, Zambianchi F, Catani F. Simple and comminuted displaced olecranon fractures: a clinical comparison between tension band wiring and plate fixation techniques. Arch Orthop Trauma Surg. 2014;134(8):1107-14.

14. Duckworth AD, Clement ND, White TO, CourtBrown CM, McQueen MM. Plate versus TensionBand Wire Fixation for Olecranon Fractures: A Prospective Randomized Trial. J Bone Joint Surg Am. 2017;99(15):1261-73.

15. Yi-Ming Ren et al. Efficacy and safety of tension band wiring versus plate fixation in olecranon fractures: a systematic review and meta-analysis. Ren et al. J Orthop Sur Res. 2016;11:137.

16. Newman SDS, Mauffrey C, Krikler S. Olecranon fractures. Injury, Int. J. Care Injured .2009; 575-81.

17. Chan KW, Donnelly KJ. Does K-wire position in tension band wiring of olecranon fractures affect its complications and removal of metal rate? J Orthop. 2014;12(2):111-7.

Cite this article as: Yogi LK, Mahi G, Thorat CR, Naik MJ. Best technique for fixation of displaced transverse olecranon fractures: tension band wiring or plate fixation? Int J Res Orthop 2020;6:1012-5. 